\title{
Laura García Sánchez
}

\section{BARCELONA, 1802. MANUSCRITOS DE LA BIBLIOTECA PALATINA DE PARMA Y SU VISIÓN ARTÍSTICA DE LA CIUDAD}

Gracias a las descripciones de ciudades que antiguos viajeros han dejado escritas en numerosos manuscritos u otro tipo de fuentes literarias, los historiadores del arte tenemos hoy en día la posibilidad de recuperar la imagen de una urbe concreta en una fecha determinada. Muchas bibliotecas y centros de investigación de todo el mundo atesoran valiosos conjuntos que permiten indagar en el pasado y comparar con el presente un amplio repertorio de monumentos, edificios, construcciones efímeras o reformas urbanísticas que, en su momento, no solamente tuvieron una gran repercusión en el inmediato entorno sino que abrieron paso a iniciativas futuras. Lugares que, por uno u otro motivo, han sido protagonistas de episodios singulares que han marcado su trayectoria tanto artística como histórica. Desde este punto de vista, Barcelona se sitúa como uno de los enclaves que mejor han protagonizado este papel, convertida en numerosas ocasiones en lugar privilegiado de acontecimientos que han dejado su sello para siempre.

La visita y larga estancia en la Ciudad Condal protagonizada por Carlos IV y María Luisa de Parma en otoño de 1802 constituye uno de esos momentos emblemáticos en los que una ciudad se transforma en escenario de un evento vinculado, en este caso, a la monarquía borbónica. Barcelona fue por entonces la ciudad escogida, tanto por su situación geográfica como por su significación histórica, para celebrar los dobles enlaces del príncipe de Asturias Fernando - el futuro Fernando VII - y su hermana la infanta Isabel con - respectivamente - los príncipes de Nápoles María Antonia y Francisco Genaro, hijos de Fernando IV - hermano de Carlos IV - y de María Carolina. El parentesco de los contrayentes invitó a concertar los enlaces, ya que eran primos entre sí, aunque fue necesaria la preceptiva dispensa del papa Pío VII. Todo quedó pactado entre la reina María Luisa de Parma y el duque de Santo Teodoro, representante de los napolitanos, el 14 de abril del 
${ }^{1}$ Calaix de Sastre es el título genérico de los setenta y tres volúmenes escritos a lo largo de cincuenta años (1769-1819) por Rafael d'Amat i de Cortada, primer barón de Maldà, quien con gran paciencia e ironía detalló todos aquellos acontecimientos barceloneses que motivaron su interés o despertaron simplemente su curiosidad. Es tanta y variada la información aportada por sus manuscritos que han sido una fuente de primera mano consultada por diversos historiadores a fin de reconstruir aspectos puntuales desde el punto de vista de un aristócrata crítico a menudo con su entorno.

2 José Coroleu, Memorias de un menestral de Barcelona 1792-1864, Barcelona, Tipografía de La Vanguardia, 1901. mismo año. Las bodas se celebrarían por poderes en julio y la ratificación e intercambio de cónyuges tendría lugar en otoño.

La presencia de los Borbones en un episodio de estas características, unida al hecho de que la familia se encontrase diversificada por entonces entre España e Italia, explica la circunstancia del por qué una variada y numerosa documentación relativa al mismo se encuentre en importantes archivos y bibliotecas italianas, especialmente de Parma, ciudad de origen de la reina María Luisa. Por su linaje y tradición, sin excluir obviamente la riqueza de sus fondos, la Biblioteca Palatina de Parma constituye uno de aquellos lugares de irresistible atractivo, especialmente cuando el motivo de estudio para un investigador está relacionado con España y los Borbones. El centro debe su fundación al interés del duque Felipe, a la voluntad política del ministro reformista Guillaume Du Tillot y a la brillante obra del bibliotecario Paolo Maria Paciaudi, religioso teatino de origen piamontés, quienes unieron sus esfuerzos con la intención común de dotar al ducado de una biblioteca de utilidad pública capaz de satisfacer cualquier tipo de estudio. Recuperar la riqueza cultural perdida desde el momento en que la reina Isabel de Farnesio pensó en su hijo Carlos como la persona adecuada para asumir el reinado de Nápoles era, por entonces, el auténtico objetivo. No contento con el nuevo desafío, Carlos decidió transferir a su nueva ciudad la librería de los Farnesio, su importante archivo y todos los tesoros artísticos, dejando a Parma huérfana de su anterior riqueza bibliográfica.

\section{Presencia regia en Barcelona}

Tras confirmarse la noticia, cuyo primer aviso comenzó a hacerse notorio en la ciudad hacia el mes de febrero de 1802, comenzaron los preparativos. Barcelona tenía que disponerse a recibir con gran dignidad y nobleza a los monarcas y príncipes, a las respectivas comitivas y al gran número de personas que vendrían a la ciudad atraídas por el esplendor de las fiestas. Mascaradas, cabalgatas, banquetes, bailes, fuegos artificiales, o conciertos proporcionaron durante un tiempo un vistoso engalanamiento, asumido en colectividad. La implicación en la visita real unió en un mismo objetivo tanto a las instituciones oficiales como a la anónima aportación ciudadana. El testimonio del sentir de esta última se encuentra en dos memorias de la época: la del barón de Maldà, ${ }^{1}$ autor del alegre panorama de una Barcelona centrada en un ambiente de gran expectación y plenamente volcada en los preparativos; y las de José Coroleu, ${ }^{2}$ quien hizo gala de una narración que más bien rayaba lo pesimista. 
Como bien indica Pérez Samper, ${ }^{3}$ la visita de los reyes a Barcelona fue una noticia que sacudió profundamente la vida de la ciudad, desde los sectores sociales más altos a los más bajos. Para la nobleza, la venida de la corte era evidentemente un acontecimiento de primer orden porque la presencia de los monarcas equivalía a grandes fiestas y a relacionarse con toda la clase alta venida de Madrid. Significaba, además, una excelente excusa para huir de la monotonía diaria. Sin embargo, también existía cierto descontento ante los cuantiosos gastos que todo ello representaba y las molestias que les iba a ocasionar la obligación impuesta de alojar en su casa a algún que otro forastero. Respecto a la clase trabajadora, la estancia de Carlos IV y María Luisa de Parma significaba, en general, más trabajo y mejor pagado. En cambio, los gremios mostraron su disgusto por el alza de sus cuotas y entre los campesinos las quejas fueron también frecuentes por los embargos a los que fueron sometidos. La primera autoridad religiosa de la ciudad, el obispo Pedro Díaz de Valdés, también mostró su satisfacción por la decisión de la corona y colaboró cuanto pudo.

Pero, a pesar de todo, parece que el común denominador fue la alegría. Al menos había dos elementos de la sociedad que esperaban con ansia tan notoria llegada. Por un lado, el juvenil, pues la estancia de la familia real implicaba fiestas, bailes, disfraces y toda clase de diversiones en un ambiente de mayor libertad que el de costumbre; y, por otro, el componente femenino, para quien la visita se traducía en fiestas, vestidos y lucimiento, especialmente entre las damas de la nobleza que tendrían que asistir a los besamanos y recepciones de palacio.

Una de las crónicas foráneas que mejor relatan el ambiente festivo de este episodio es el manuscrito del prior Michele Colombo, ${ }^{4}$ quien se encontraba en Madrid justo en el momento en que tuvo lugar la celebración del matrimonio por poderes de la infanta María Isabel con el príncipe de Nápoles. ${ }^{5}$ Según narra este personaje:

Io sono capitato in Madrid pochi giorni prima che si solemnizzassero gli sponsali dell'Infanta $D^{a}$. $M^{a}$. Isabella figlia dell LL.MM Cattoliche col Principe ereditario delle due Sicilie. Ci fu galla a tal effetto alla Corte per tre giorni: ció fu a' 6,7, e 8 di Luglio. La mattina de' 6 L'Ambasciator estraordinairio di Napoli fece il suo solemne ingresso per La consueta formalità di chiedere solemnemente I'Infanta $D^{a}$. $M^{a}$ Isabella per esposa del Primogenito del Re delle due Sicilie (N'erano già stati conclusi gli sponsali molti mesi prima in Aranjuez unitamte. a quelli del Principe delle Asturie con una delle fliglie del detto Re delle due Sicilie). Quest'Ingresso fu magnifico. Nel di seguente il Re e la Regina ammisero i cavalieri e Le Dame al bacio della mano. Agli 8 la mattina fuerono ammessi al bacio della mano I Consigli; en nel dopo pranzo si portanno Le Loro Maestà im gran ceremonia alla Cappella Reale nella
3 María de los Ángeles PÉRez SAMPER, Barcelona, Corte. La visita de Carlos IV en 1802, Barcelona, Publicaciones de la Cátedra de Historia General de España, 1973.

4 El prior Michele Colombo (Campo di Pietra, 1747 - Parma, 1838) alcanzó un notable prestigio como educador entre las familias nobles italianas, lo que le llevó a frecuentar y vivir en diversas ciudades. El carisma conseguido en Padua le condujo a ser llamado a Parma en 1796 como instructor del joven Giovanni Buenaventura Porta, heredero de un notable patrimonio. Casa Porta fue para Colombo su definitiva vivienda y lugar donde pasó cuarenta años, algunos de ellos viajando, primero en calidad de maestro itinerante y posteriormente como fraterno amigo de su otrora discípulo Giovanni. En julio de 1802 se encontraban ambos en BarceIona, procedentes de Madrid, donde participaron en las fiestas de gala en ocasión del doble matrimonio de los príncipes. Los dos viajeros Ilevaban cartas de presentación del duque de Parma, Fernando de Borbón y hermano de la reina María Luisa, para los monarcas españoles. De aquí el relato de sus viajes en éste y otros manuscritos, al margen de su epistolario privado.

5 Nicoletta AgAzzI, Intorno all'abate Michele Colombo, Vittorio Veneto, Kellermann Editore, 1995. 
6 Michele Colombo, manuscrito parmense 3789, pp. 112119. Parma, Biblioteca Palatina.
Chiesa di Nuestra Señora de Atocha ad assistire al Tedeum in ringraziamento de'sopradd. Sponsali. La pompa fue piena di dignità. Tutti i palazzi e Le case sulla via per la quale pasar dovevano Le MM.LL. erano addobbate qual più riccamente e qual meno secondo La possibilità di quelli che le abitavano. Due file di soldati dove di cavalleria e dove di fantasia erano appostate lungo La strada per tener in dovere La folla del popolo, che era inmensa. "L'accompagnamento delle Loro Maestà fu, secondo il costume, in questa maniera*: [...]» Fuvoi illuminazione per tres ere al Real Palazzo, alla Casa dell'Comune, a qualche di alcuni Grandi si Spagna, de'Ministri, ed ad altre. La terza será fu illuminato lo stradone dalla Chiesa di Atocha fino al Corso con Lumi disposti a pirámide, all' uso di Parigi, che presentavano un colpo d'occhio bellissimo: illuminate furono pure la calle-de-Atocha, la Plaza-mayor, la calle-nueva, la calle-mayor, per cui si restituirono le LL.MM. a Palazzo. La illuminazione della Piazza maggiore, fatta a spese de los cinco Gremios; quella del palazzo della Compagnia de los cinco Gremios, quella delle Case della Compagnia de Las Filipinas, e quella del Corregidor attraevano particularmente a se gli occhi. Il Governo aveva preso, como ho annunziato di sopra, la prevenzione opportune perchè in una folla tanto inmensa di popolo fose mantenuto il buon ordine; ma la urbanità degli abitanti di Madrid non diede la minima cagione alla polizia di essercitarsi la sua autorità. ${ }^{6}$

\section{Reformas realizadas}

Uno de los aspectos que más preocupó al Ayuntamiento fue el modo en el que recibir adecuadamente a todas las familias reales que eran esperadas. Barcelona no debía resolver solamente las expectativas centradas en la figura de Carlos IV y María Luisa de Parma, acompañados de sus hijos, sino también de los reyes y príncipes napolitanos. A éstos cabía añadir la presencia de los reyes de Etruria (antiguo Gran Ducado de Toscana), un joven matrimonio formado por María Luisa de Borbón - hija de los monarcas españoles - y por su primo, el príncipe Luis - hijo del duque Fernando de Parma - , carente de ambición y personalidad para llevar las riendas de un estado.

El problema más generalizado fue que los responsables de la preparación de la ciudad, así como del recibimiento y de los obsequios, no disponían de antecedentes en situaciones parecidas sobre los que orientarse. La precedente visita real, la de Carlos III en 1759, había tenido como protagonista a una única familia real. En el caso de Carlos IV, el problema se dimensionó en todos los aspectos, no tan sólo por la singularidad de la reunión en Barcelona de tantas personalidades de la realeza, sino también por la numerosa comitiva que traía consigo: la corte, los criados, los guar- 
dias de corps... Aparte de los directamente vinculados a las comitivas reales, había que contar con el señalado gentío que, procedente de toda la provincia e incluso del resto de España, se iba a dar cita en la ciudad atraída por la posibilidad de ver a sus reyes y por las fiestas organizadas. Las autoridades correspondientes tuvieron que afrontar un sinfín de cuestiones a fin de hacer cómoda y sin problemas la estancia de tan particulares huéspedes. De entre éstas sobresale, sin duda alguna, la decisión de los alojamientos.

Sin embargo, queda fuera de toda duda que uno de los aspectos más relevantes de la venida real fue la gran reforma urbana e infraestructural realizada. Incluyó aspectos como la mejora y el empedrado de las calles, la pavimentación y el problema de los mostradores de venta de la calle de la Platería, el tema de los carruajes, la renovación de las casas y el ensanche y alineación de las calles, la cuestión del alcantarillado, la mejora de las conducciones y el abastecimiento de agua, la limpieza de la ciudad y la de las fachadas o las prevenciones en caso de incendio. A todo ello cabe añadir la conclusión de obras magnas, como la Lonja o el Laberinto de Horta; la ampliación del Teatro Principal; la reforma del Palacio Real y del puente que lo unía a la tribuna de la iglesia de Santa María del Mar; las decoraciones y los montajes escenográficos efímeros, pensados para cubrir los edificios más representativos; o los monumentos conmemorativos destinados a perdurar en la memoria y en el tiempo.

El núcleo se centró en la plaza de Palacio, centro político y económico de la ciudad desde finales del siglo XVIII. La importancia de este enclave no pasó desapercibida a ojos de los viajeros extranjeros y visitantes de otras ciudades ya que conocían de antemano la disposición del consistorio de alojar a los monarcas españoles en el Palacio Real, a la familia napolitana y a los reyes de Etruria en el palacio de la Aduana Nueva y a Manuel Godoy y su séquito en la Casa Lonja. En resumen, edificios todos ellos importantes en el actualmente denominado Pla de Palau y de los que tan sólo ha desaparecido el primero.

\section{La narración de Guglielmo Fontebuoni}

En relación con la estancia real, la Biblioteca Palatina de Parma conserva, al margen del manuscrito ya citado del prior Michele Colombo, tres manuscritos fundamentales. El primero de ellos, redactado por Gugliemo Fontebuoni, se titula Viaggio in Spagna Delle LL. Maestà Il Re e la Regina di Etruria Lodovico Primo e Maria Luisa Infanti di Spagna che ebbe luogo dal di 28 Settembre 1802 al 7 Gennajo 1803 con le notizie Geografiche, 
7 Biblioteca Palatina de Parma, manuscrito palatino 426.

8 El manuscrito con las memorias se conserva en el Archivo Borbónico de la Orden Constantiniana de San Jorge, también en Parma.

9 Guglielmo Fontebuoni, manuscrito palatino 426, 1844, p. 50, Parma, Biblioteca Palatina.
Storiche, \&\&, di tutti i Paesi percorsi dalla Reale Comitiva. ${ }^{7}$ Constituye una de las fuentes más importantes relativas al encuentro entre los reyes y príncipes en Barcelona. A pesar de que está centrado principalmente en la narración de los preparativos, el viaje, la estancia, la visita a otros lugares de Cataluña y el posterior regreso a Florencia de los reyes de Etruria, se trata de una obra igualmente significativa en lo concerniente a las descripciones de las que sobre la ciudad, el acontecimiento real y las fiestas celebradas hace gala su autor. Dicho personaje, cuyas primeras líneas escritas a modo de introducción permiten interpretar que se trataba de un miembro del séquito de María Luisa de Borbón desplazado a Barcelona, dedicó su obra, fechada en 1844, a Carlos Luis, primogénito de los reyes de Etruria. Aunque tan sólo contaba con siete años de edad cuando tuvo lugar este importante acontecimiento entre las familias borbónicas, el destino le tenía reservado, tras el fallecimiento de su madre, la regencia del ducado de Luca (fig. 1).

La narración de Fontebuoni no se basa solamente en los recuerdos personales de lo visto y vivido en Barcelona, sino que parte de su relato se apoya en cartas y documentos pertenecientes a otras personalidades también desplazadas en el viaje y que, con el tiempo, llegaron a sus manos. La rigurosidad de lo escrito, al margen de algunos comentarios realizados en cierto tono jocoso pero no por ello exentos de veracidad, hacen de su obra una fuente documental comparable en cierta manera por el estilo y las anécdotas descritas al ya citado Calaix de Sastre del barón de Maldà. Dado que existen otras lecturas más modernas relativas al desplazamiento de los reyes de Etruria a Barcelona, pero todas ellas impresas, esta circunstancia corrobora aún más si cabe la importancia, por su escasez, de las fuentes manuscritas citadas, a las que hay que añadir las memorias escritas por la propia María Luisa de Borbón. ${ }^{8}$

A título de ejemplo, y a nivel urbanístico, uno de los edificios que más llamó la atención del cronista fue, precisamente, el de la Lonja, citado en su texto como el «Palazzo della Borsa, abitato nel primo piano dal Principe della Pace e dalla Scuola delle Belle Arti situata al secondo piano di detto Palazzo». ${ }^{9}$ Por otro lado, no sorprende en absoluto que despertara su curiosidad, ya que fue una de las construcciones emblemáticas de Barcelona finalizadas tanto en su parte constructiva como decorativa con ocasión de la visita real.
1. Parma, Biblioteca Palatina, portada del manuscrito palatino 426, 1844. Su extenso título puntualiza con rigurosidad la información desarrollada en la narración interior. 
Questo palazzo ocupa il piú bel posto di Barcellona, giacché risiede al principio di una bellissima spianata che guarda il mare e che presenta una curva parabolica non dissimil da quella del Palladio, ricca di statue, e tutte incrostate di una pietra calcárea bigia che si cava nel vicino Monte Govi, e che resiste passabilmente all'aria. La principale di dette facciate che presenta l'ingresso alla gran Borsa, rimane sopra la piazza principale di Barcellona in faccia al palazzo del Vice-Re, e le altre due assai piú prolungate guardano la prima il mare, e l'altra una strada o calle delle meno anguste di quella citta.

Al terreno di questo palazzo vie era la Borsa, il primo piano era abitato dal Principe della Pace e al secondo eravi la Scuola delle Belle Arti (figs. 2 y 3).

La gran sala era destinata per la Borsa ed è divisa in tre navate sostenute da tre soli archi sul gusto seracenico, o gotico, se si eccettui la curva molto dolce, ma con arco scorniciato a bastone rotondo in luogo di essere piano. Le colonne altresi destínate a sostenere questi maestosi archi sono piuttosto sottili, altissime, montate da un piccolo capitello e scannellate a bastoni. Una magnifica ringhiera con parapetto ornato di bronzi dorati, circonda da ogni parte questa sala e la rende capace di serviré a delle feste di ballo giacché il di lui parapetto presenta di tratto in tratto dei viticci per situarvi i torcetti.

Nel primo piano del palazzo abitava il Principe della Pace quale soleva dare la sua udienza nel pasarse dal propio quartiere a quello dei segretari.

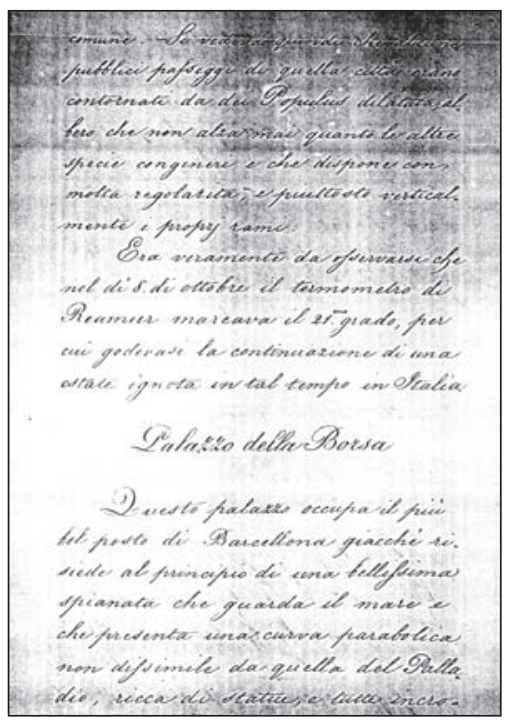

2. Parma, Biblioteca Palatina, manuscrito palatino 426, 1844, p. 52. La riqueza de este fragmento en concreto reside, en parte, en la descripción de Fontebuoni acerca de la ubicación del edificio y su entorno.

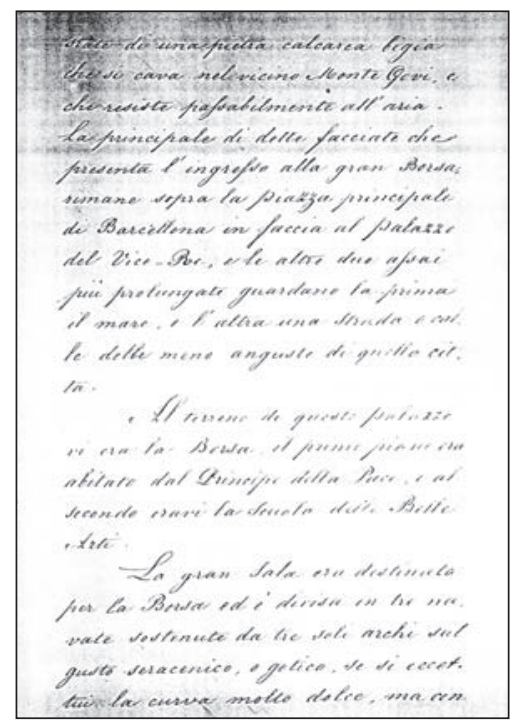

3. Parma, Biblioteca Palatina, manuscrito palatino 426, 1844, p. 53. El emplazamiento de la Casa Lonja despertó la admiración del autor, quien se entretuvo en mencionar incluso las diferentes fachadas del edificio. 
Nella mattina del 9 ottobre appunto si vedevano ivi radunate piú di trecento persone di tutti i ceti, escluso quello dei plebei, le quali a doppie file e stando in piede circondavano le pareti di tutte quelle camere per le quali era obbligato a passare il Sig, Principe che rappresentando, e all' apparenza e col fatto, il vero Monarca delle Spagne, ascoltava pasando le diverse suppliche di ognuno totalmente un pubblico, nel tempo che i suoi segretari, che le seguivano andavano raccogliendo ciascuno memoriale: alla pari con tutti gli altri vi erano tre prelati in mantelletta e molte Gran Croce di diversi ordini (figs. 4 y 5).

Il secondo piano di questo maestoso palazzo era tutto quanto dedicato alla Scuola delle Belle Arti, e per tale oggettto trovavasi subdiviso in vaste e luminose gallerie, alle pareti delle quali oltre i banchi per gli scuolari si vedevano appese i migliori disegni, stampe e gessi, e tuttociò che poteva abbisognare per l'istruzione dei giovani, tanto per il disegno di figura quanto per I'ornato. Gli originali per gli elementi del dissegno erano dappertutto quegli stessi pubblicati dal Morghen Professore d'Incisione nell'Academia delle Belli Arti di Firenze, di cui ivi si vedevano le migliori prove delle su estampe unite a quelle di altri valenti incisori. Si vedevano inoltre due gallerie, una delle statue piú celebri in gesso e l'altra di quadri piú classici copiati a olio da diversi pensionati in Roma, e specialmente da un certo Rodriguez e da un

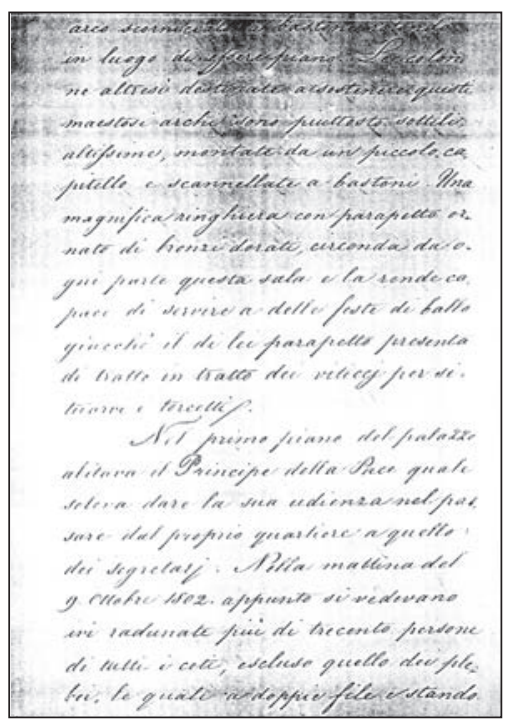

4. Parma, Biblioteca Palatina, manuscrito palatino 426,1844 , p. 54 . La joya arquitectónica de la Lonja, su salón gótico, no pasó desapercibido al cronista. Al margen de describirlo, explica también su eventual conversión en sala de baile.

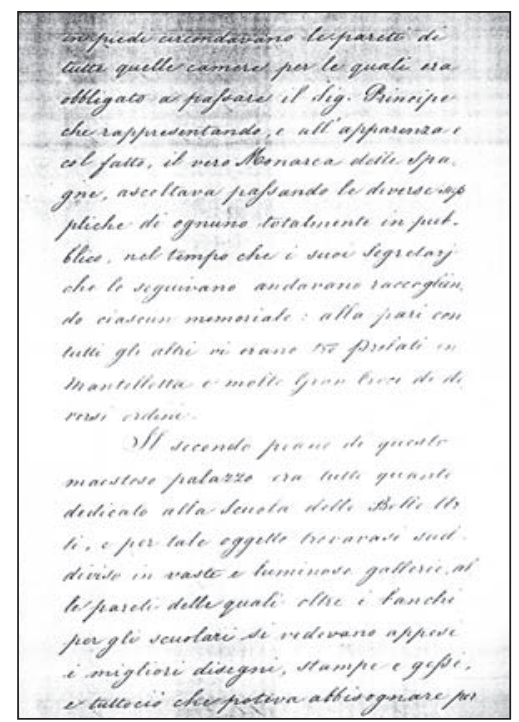

5. Parma, Biblioteca Palatina, manuscrito palatino 426, 1844, p. 55. El hecho de que el primer piso de la Casa Lonja fuese acondicionado como residencia del ministro Manuel Godoy implicó muchas mejoras para éste y otros usos vinculados a su cargo. 
certo Montagna. Finalmente si conservava in una stanza separata un superbo catafalco per la statua di Gesú morto, che si esponeva nella Chiesa primaziale nel venerdí santo, tutto di argento sodo ben lavorato con rapporti dorati (fig. 6)..$^{10}$

Resulta curiosa la descripción realizada por el autor no tan sólo del emplazamiento urbanístico, del magnífico salón gótico del edificio, de las audiencias concedidas por Manuel Godoy o de la Escuela de Bellas Artes, sino por la citación de nombres como los del pintor Francesc Rodriguez Pusat (1767-1840) y, especialmente, de Pere Pau Montanya (1749-1803). Este último, sucesor en la dirección de la Escuela Gratuita de Dibujo _ instituida en la propia Lonja gracias a la denominada Junta de Comercio - del grabador Pasqual Pere Moles, ${ }^{11}$ no se erigió tan sólo como el responsable del programa escultórico que ornamentó especialmente el exterior de la Casa Lonja, pensado en exclusiva para los reyes, sino que fue también el artífice de gran parte de las decoraciones, iluminaciones y carrozas realizadas para conmemorar el evento.

Otro de los lugares destacados por Fontebuoni es el antiguo paseo de la Explanada, conocido actualmente como paseo de Sant Joan, erigido bajo la dirección del Capitán General Agustín de Lancaster. Iniciado el 16 de agosto de 1797, la idea de este amplio bulevard no hizo sino continuar una serie de mejoras urbanísticas afrontadas por la ciudad en los años inmediatamente anteriores a 1802, encabezadas por la Rambla y la urbanización de la BarceIona nueva, empezada en 1787 con el plan de parcelación del Raval.

La venida de los monarcas a la ciudad fue una excelente excusa para mejorar y embellecer un paseo relativamente nuevo y punto de encuentro de los ciudadanos. Hacia la parte de mar se realizó un empedrado semicircular y el resto se dejó con arena a fin de que los numerosos carruajes de la corte pudieran circular sin problemas. Ante la indignación de los barceloneses, que los detestaban, ${ }^{12}$ los ingenieros milita-

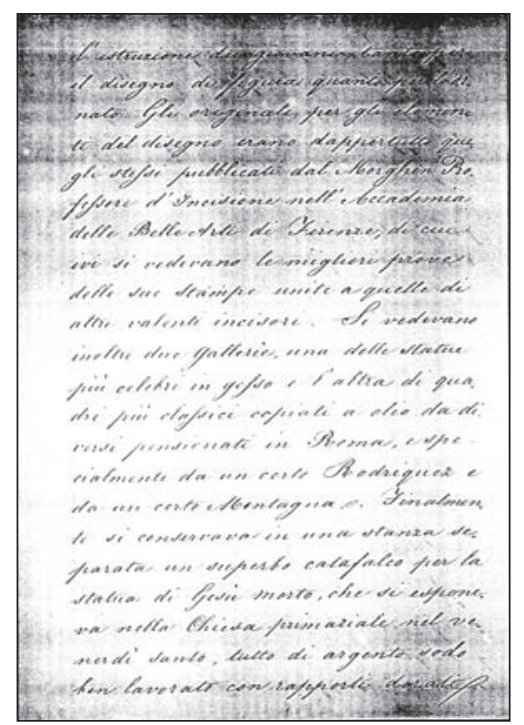
palatino 426, 1844, p. 56. La Escuela de Bellas Artes, ubicada entonces en el segundo piso del edificio, mereció también la atención de Fontebuoni, atraído por la gran cantidad de obras expuestas de estudiantes y docentes.
6. Parma, Biblioteca Palatina, manuscrito
10 Guglielmo Fontebuoni, manuscrito palatino 426, 1844, pp. 52-56. Parma, Biblioteca Palatina.

11 Rosa María Subirana Rebull: «Pasqual Pere Moles, primer director de l'Escola Gratuïta de Dibuix», D'Art, 12, 1986, pp. 173-180. De la misma autora, Pasqual Pere Moles i Corones (València, 1741 - Barcelona, 1797), Barcelona, Biblioteca de Catalunya, 1999.

12 Rafael d'AMAT I DE CORTADA (barón de Maldà), Calaix de Sastre, vol. Xxv, 19 de junio de 1802. 
13 Carlos Cid Priego: «El arte barcelonés y las visitas reales de 1802», Hispania, Revista Española de Historia, Madrid, CSIC, tomo Xv, núm. LIX, 1955, pp. 231-285.

14 Rosa Maria Garcia DoMÈNECH, El passeig de l'Esplanada o de Sant Joan (17971874), Barcelona, tesis de licenciatura inédita, 1981. res demolieron las fuentes de los dos extremos de la parte central del paseo para poner otras de mejores, circunstancia que convirtió el lugar en centro de reunión de los más escépticos. Sin embargo, no tardaron en cambiar de opinión cuando las nuevas fuentes empezaron a tomar forma. Los comentarios despectivos fueron rápidamente sustituidos por muestras de admiración en el momento que vieron las esculturas destinadas para su adorno, realizadas por artistas tan importantes en la época como Salvador Gurri. ${ }^{13}$ Una cuidadosa selección del arbolado, bancos y otros surtidores en los puntos intermedios contribuyeron a dotar de gran belleza el conjunto.

Dado que fue un paseo que tuvo una dilatada construcción y renovación a lo largo del tiempo, ${ }^{14}$ el texto de Fontebuoni resulta esencial para saber cómo era realmente en la precisa fecha del año 1802. Así, relató al respecto:

Questo pubblico passeggio consisteva in cinque viali affatto in piano e paralleli fra loro, due dei quali i più estesi servivano per le carrozze ed i tre interni per le persone a piedi. La lunghezza dei viali era quasi di un miglio. Agli estremi punti del viale di mezzo s'incontravano due magnifici gruppi di statue con fontane di acqua viva e nel mezzo dello stesso viale si vedevano due vasche ovali contornate da ringhiera di ferro ed ornate di fontane esse pure. I viali suddetti erano separati fra loro da filasi di alberi irrigati ciscuno al propio pedale da un rivolo di acqua corrente, che derivava dall'avanzo di tali vasche o fontane. Fra un albero e l'altro era situata una panchina di pietra colla respettiva spalliera ad uso di piccola ringhiera di pietra.

Le vasche e gruppi di fontane erano circondati di salci piangenti (salix babylonia Linn), e gli alberi che cincordavano i viali appartenevano alle seguenti specie: Populus dilatata - Populus tremula - Olmus campestris parifolia - Alcer pseudo platanus; ma non essendo tali alberi della natura di quelli che nascono attorno ai fiumi non potevano generalmente prosperare attesa la continua irrigazione. Si osservava in fatti a colpo d'occhio pregiudicato nella propia vegetazione, e talvolta affatto perduto ognuno di detti alberi non destinato dalla natura a vivere nell'acqua. Meno quest'errore geoponico, ben facile a rimediarsi col tempo, il passeggio era veramente delizioso, e lo diveniva ancora piú della sera per essere illuminato da fanali e decorato da due Bande militari che suonavano alternativamente della buona musica. Il concorso di tutti i ceti era sempre grande, ed il bel sesso pure in gran numero: ma le fisonomie amabili erano molto rare in Barcellona, ed essendo vere. restavano eclipsate dai loro abiti sempre neri e da una velatura bianca che le rendeva simili alle monache. Si rendeva poi particolare il vedere sempre queste donne accompagnate con' preti vestiti di lungo con grandissimo cappello spuntato in testa, o sivvero con i frati di tutti gli ordini possibili, ai quali era licito anco di andare al teatro fino alla mezza notte. ${ }^{15}$ 
Cerca del paseo de la Explanada, Fontebuoni quedó también sorprendido por el recinto de la Ciudadela, dentro de las murallas de la ciudad, a la que describió como una auténtica fortaleza que

[...] cinta da un doppio larghissimo fosso e presentando da ogni parte dei baluardi e fortificazioni si rende quasi inespugnabile. Le due parte di terra e di mare sono di una massiccia ben'intesa architettura. La Piazza d'Arme è assai spaziosa di forma quadrata e circondanta all'intorno da quartieri e caserme militari; è altresi provvista di due buone e abbondanti fontane che traggono origine dalle montagna che la circondano da NO. In disparte evvi una altissima torre che presenta un minaré turco e conserva degli ornati di terra cotta del suo colore da potere argomentare che sia un avanzo seraceno». ${ }^{16}$

\section{EI Viage que SS. MM. hicieron a Barcelona y el regreso al Real Sitio de Aranjuez}

Al margen de la plaza de Palacio y su entorno, Fontebuoni halaga en su manuscrito la actividad manufacturera y comercial de Barcelona, es decir, la presencia de fábricas y todo aquello que tuviera relación con la industria, aspecto que tuvo su incidencia en la organización de la visita real al intervenir en aspectos puntuales de la misma. En las tareas de preparación y control, la colaboración del Cuerpo de Colegios y Gremios y del de Comercio y Fábricas resultó poco menos que indispensable.

Distintas son, sin embargo, las prioridades del relato de los otros dos manuscritos que, sobre este tema, conserva la Biblioteca Palatina de Parma. Al Viage que SS. MM. hicieron a Barcelona saliendo de Madrid el día 12 de Agosto de 1802 se une el Viage que SS. MM. hicieron volviendo de Barcelona al Real Sitio de Aranjuez a donde llegaron el día 8 de Enero de $1803 .{ }^{17}$ De autor desconocido, la riqueza descriptiva de ambos textos resulta incuestionable no ya tan sólo en el ámbito de los pueblos y ciudades por las que pasaron los monarcas y la relación de sus edificios y monumentos más destacados, sino porque el texto incide también en costumbres, población, formas de vida, oficios, etc. (figs. 7 y 8 ).

En el caso de Barcelona, destaca que su población era en aquel momento de 74.770 personas,

[...] sin incluirse en este numero los Párvulos, los individuos del Clero Secular y Regular, el muy considerable de personas sugetas a la jurisdicción castrense y el de estrangeros y transeúntes que no constan en las Matrículas Parroquiales
15 Guglielmo Fontebuoni, manuscrito palatino 426, 1844, pp. 57-59. Parma, Biblioteca Palatina.

16 Guglielmo FonteBuoni, manuscrito palatino 426, 1844, pp. 60-61. Parma, Biblioteca Palatina.

17 Biblioteca Palatina de Parma, manuscrito palatino 863 y 864 (vols. I y II). 
18 Viage que SS. MM. hicieron a Barcelona saliendo de Madrid el dia 12 de Agosto de 1802, manuscrito palatino 863, Parma, Biblioteca Palatina.

lo cual, unido a las 2.326 personas que, según el «plan de aposentamiento» trazado por las autoridades de Madrid, formaban parte de la comitiva regia española, permite aproximarse al número total de las que llegaron a reunirse con motivo de la visita real en la Ciudad Condal. Así, el anónimo cronista subraya:

[...] no es fácil figurarse el bullicioso tragino de Estrangeros y Nacionales que concurren en su Puerto, esperando unos sus mercancías y otros proporcionando la fácil salida de las mismas, ni el cuydadoso esmero con que esta gente laboriosa anda por sus intereses, en su propio veneficio, y veneficio del estado procurando cada individuo en sus respectivos Oficios y Artes imitar y adelantar a los estrangeros. ${ }^{18}$

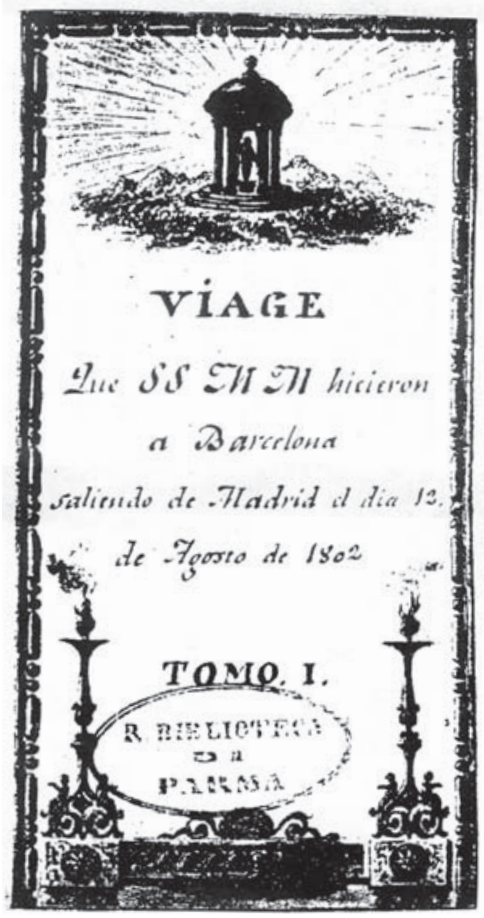

7. Parma, Biblioteca Palatina, frontispicio del manuscrito palatino 863. Sin paginar, el final del tomo ofrece un interesante resumen de la distancia recorrida por los monarcas en leguas y el número de horas invertidas en el total del desplazamiento.

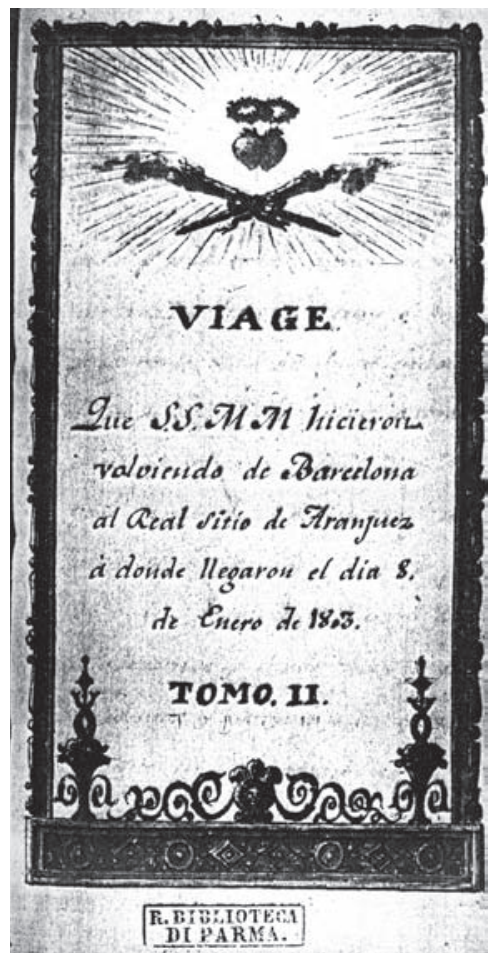

8. Parma, Biblioteca Palatina, frontispicio del manuscrito palatino 864. De características similares al anterior, la narración comienza el día 8 de noviembre de 1802, fecha en la que Carlos IV y María Luisa de Parma emprendieron el regreso hacia Aranjuez. 


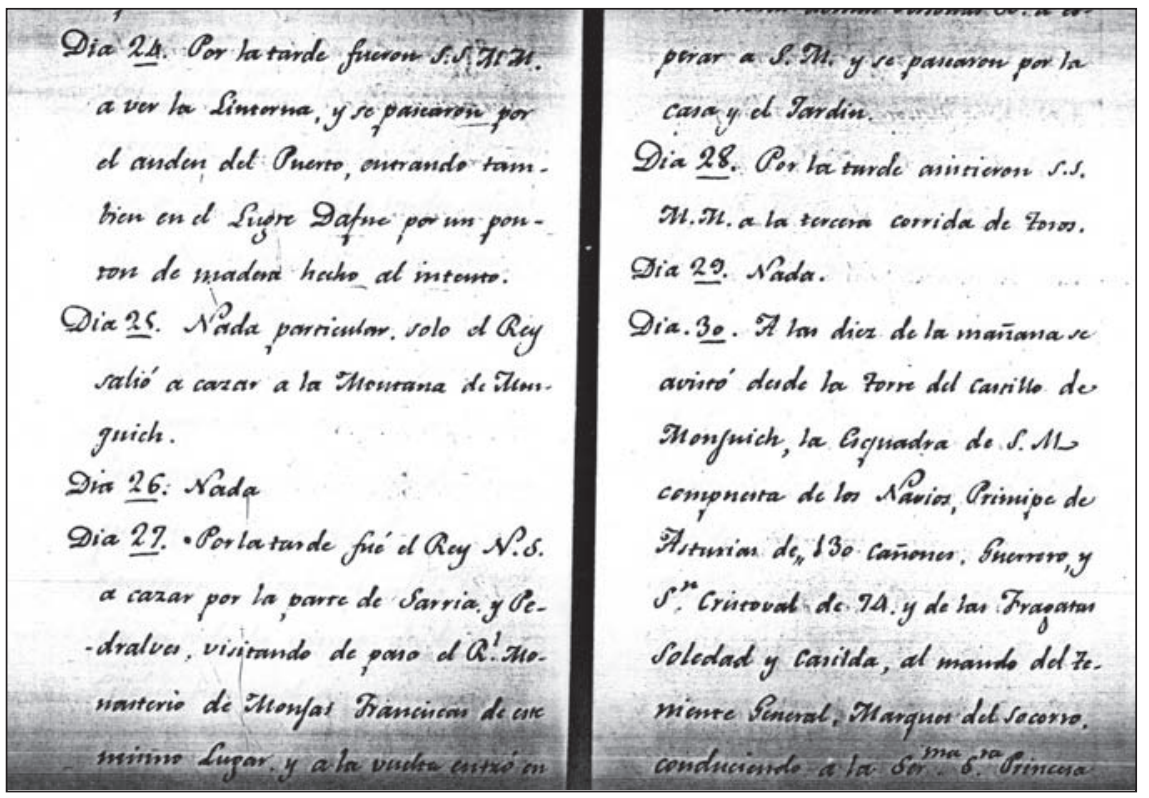

9. Parma, Biblioteca Palatina, manuscrito palatino 863. Los días indicados corresponden a las actividades realizadas por los monarcas del 23 al 30 de septiembre, circunstancia que permite reconstruir la cotidianidad de los ilustres huéspedes.

A continuación, describe las parroquias más importantes de la ciudad así como los conventos, citados en número de 12; los clérigos regulares; los monasterios de religiosas; las iglesias particulares; los colegios de regulares; las capillas públicas; la composición del Estado Militar, de la Plaza, de la Ciudadela, de la Comandancia de Marina, de la Capitanía del Puerto, de la Contaduría de Marina, de los Tribunales, de la Casa de la Ciudad, de la Real Tabla de Cambios, de la Contaduría del Ejército, de la Tesorería del Ejército y de la Administración de Aduanas. A nivel urbanístico y cultural destaca las puertas de la ciudad; las Casas de Instrucción (es decir, Reales Academias y el Real Colegio de Cirugía, con la puntualización de la Escuela de Náutica y de Bellas Artes de la Lonja) y recoge la ubicación de las bibliotecas públicas. Cierra todo este conjunto la enumeración e interesante - aunque breve- descripción de edificios notables, entre los cuales, curiosamente y a diferencia de Fontebuoni, define a la Casa Lonja o Consulado tan sólo como «bastante bien decorada». Destaca, en cambio, la Casa de la Audiencia, las Atarazanas, la Aduana Nueva, el Palacio del General, la Ciudadela, el castillo de Montjuïch, el barrio de la Barceloneta "fuera de la Puerta del Mar» y concluye con la muralla de mar. También hay un apartado dedicado a la indus- 
tria y a la fabricación de sedas, algodón, jabón, armería y alfarería. El resto del texto está centrado en la relación diaria de las actividades de los monarcas durante todo el tiempo que permanecieron en Barcelona (fig. 9).

En resumen, este tercer manuscrito de la Biblioteca Palatina de Parma contribuye a perfilar el panorama más elaborado de Guglielmo Fontebuoni. Aunque el punto de partida o argumento es el mismo, la descripción de puntuales enclaves o edificios de la Ciudad Condal difiere en ambos, ya que no se les otorga la misma prioridad. Con todo, resultan útiles para recuperar aquella imagen de la que se hablaba en un principio, ya que gran parte de la información aportada constituye un importante eslabón a la hora de trazar un fragmento de la historia artística y urbanística de Barcelona.

Laura García Sánchez Universitat de Barcelona laura.garcia@ub.edu 
Barcelona, 1802. Manuscritos de la Biblioteca Palatina de Parma y su visión artística de LA CIUDAD

En el año 1802, Barcelona se convirtió en punto de encuentro de las familias reales española y napolitana, desplazadas para asistir al enlace matrimonial de sus hijos. La elección de la ciudad como punto de intercambio de los prometidos significó un gran desafío para las autoridades barcelonesas de entonces, que se vieron obligadas a solventar multitud de cuestiones a fin de hacer cómoda y factible la presencia de tan ilustres huéspedes. Uno de los aspectos que tuvieron que afrontar fue el de una modélica reforma urbanística a todos los niveles, desde el empedrado de las calles a problemas relacionados con la higiene de la ciudad o de la llegada del agua. La plaza de Palacio, punto de residencia de la familia real española (Palacio Real), la napolitana (Palacio de la Aduana Nueva) y del primer ministro Manuel Godoy (Casa Lonja) fue uno de los enclaves más remodelados. El resultado no fue tan sólo orgullo de los participantes, sino que quedó reflejado incluso en las impresiones escritas por visitantes foráneos y cronistas del viaje real.

Palabras clave: urbanismo, arte neoclásico, historiografía, literatura artística, arquitectura del siglo XIX, arte catalán, historia de Barcelona.

Barcelona, 1802. Manuscripts of the Court Library of Parma and his artistic Vision of the CITY

In the year 1802, Barcelona became the point of encounter of the Spanish and Neapolitan Royal families, which arrived to attend the marriage of their children. The choice of the city as the promised exchange point meant a great challenge to the Barcelona autho rities at that time, who were forced to deal with a multitude of issues in order to render the presence of such illustrious guests comfortable and workable. One of the aspects that had to be dealt with was an exemplary urban reform at all levels, from the pavement of the streets to problems related with sanitation or the availability of water. The square then called Plaza de Palacio, which housed the royal families of Spain (in the Royal Palace) and Naples (in the Palacio de la Aduana Nueva) and the Prime Minister Manuel Godoy (in the Casa Lonja) was one of the more drastically remodeled areas. The result not only made the participants proud but it was also reflected in the written impressions provided by foreign visitors and chroniclers of the royal trip.

Keywords: urbanism, neo-classical art, historiography, artistic literature, architecture of the $19^{\text {th }}$ century, Catalan art, history of Barcelona.

DOl10.1344/Materia2014.8.8 